\title{
Haldane Gap and Fractional Oscillations in Gated Josephson Arrays
}

\author{
Ehud Altman ${ }^{1}$ and Assa Auerbach ${ }^{1,2}$ \\ ${ }^{1}$ Department of Physics, Technion, Haifa 32000, Israel. \\ ${ }^{2}$ Department of Physics, Stanford University, CA 94305, USA.
}

(January 27, 2018)

\begin{abstract}
An analogy between the twisted quantum $x x z$ model and a gated Josephson junction array is used to predict sharp structure in the critical currents versus gate voltage, and fractional AC Josephson frequencies. We prove selection rules for level crossings which imply fractional periodicities of ground states with varying Aharonov-Bohm flux. Extrapolated numerical diagonalization on ladders, find a Haldane gap at moderate easy-plane anisotropy, with vanishing superfluid stiffness. Physical parameters for experimental realization of these novel effects are proposed.
\end{abstract}

74.50.+r, 75.10.Jm

Quantum phase fluctuations in superconductors can drive zero temperature superconductor to insulator transitions [1], as observed for example in disordered films [2]. Their effects can be enhanced and studied in detail using a weakly coupled, low capacitance Josephson junction array (JJA) [3: [4].

Theory of quantum phase fluctuations has used models of interacting bosons on a lattice [5], and quantum dynamics of vortices [6]. The latter approaches have proposed collective phases such as vortex Bose condensation [7] (for the insulator), and fractional Quantum Hall phases (for JJAs in magnetic field [8]).

Lattice bosons map onto effective models of quantum spins. A popular approximation to the phase diagram is mean field theory on the classical (large $S$ ) spin model [9]. In the strongly quantum regime, the same mapping relates the Mott insulator (integer bosons per site) and the quantum disordered antiferromagnet [5].

In this paper we explore the quantum magnetism analogy further. We focus our attention to the effects of a periodic lattice on superconductivity. We study the quantum xxz model with twisted boundary conditions (i.e. an Aharonov Bohm (AB) flux) both numerically and analytically. The many body spectrum, vortex tunneling rates, and superfluid stiffness are computed for different lattice dimensions and magnetization (Cooper pair density). We prove general selection rules for symmetry protected level crossings. This rule imposes fractional periodicities of the ground state as a function of $\mathrm{AB}$ flux, and is closely related to the "fractionally quantized phases" found by Oshikawa, Yamanaka and Affleck (OYA) in magnetized Heisenberg chains [10].

The following effects may be observed in JJAs of dimensions $L_{x} \times L_{y}$.

(i) Haldane gap. Our numerical results for the $x x z$ model on a two leg ladder find a Haldane gap in a sizeable regime of the easy plane anisotropy parameter. The lowest gap remains finite, while the superfluid stiffness decays exponentially for $L_{x} \rightarrow \infty$. The Haldane phase is characterized by a suppressed critical current (relative to its classical value), and a high AC Josephson frequency
$f_{Q}=2 \mathrm{eV} / \mathrm{h}$, where the classical Josephson frequency of the array is $f_{c l}=2 \mathrm{eV} /\left(h L_{x}\right)$.

(ii) Fractional Oscillations. At fractional Cooper pair densities $n=p /\left(q L_{y}\right), p, q$ integers, selection rules derived below produce sharp dips in the critical current $I_{c r}$ versus gate potential. In these states, AC Josephson oscillations appear at subharmonic frequencies $f_{Q}^{q}=f_{Q} / q$.

We conclude by proposing physical parameters for experiments.

The short range Bose Hubbard model is given by

$$
H^{B H}=U \sum_{i} n_{i}^{2}+\sum_{\langle i j\rangle}\left(V n_{i} n_{j}-2 J\left(e^{i \theta_{i j}} b_{i}^{\dagger} b_{j}+\text { H.c }\right)\right)
$$

where $b_{i}^{\dagger}$ creates a boson (Cooper pair [11]) at site $i$ on a square lattice with nearest neighbor bonds $\langle i j\rangle$, and $n_{i}=b_{i}^{\dagger} b_{i}$. The lattice is placed on a cylinder penetrated by an $\mathrm{AB}$ flux $\Phi$, introduced via the gauge phases $\theta_{i j}=$ $\delta_{j, i+\hat{x}} 2 \pi \phi / L_{x}$, where $\phi=\Phi / \Phi_{0}$, and $\Phi_{0}=h /(2 e c)$. The supercurrent in the $x$ direction is given by $I_{s}=\frac{1}{h}\left\langle\frac{\partial H}{\partial \phi}\right\rangle$.

At large $U>>J, V$ one can keep the two lowest energy Fock states at every site, say $\left|\bar{n}_{i}\right\rangle$ and $\left|\bar{n}_{i}+1\right\rangle$, and project out all other occupations. In the projected subspace, $b_{i}^{\dagger}, b_{i}, n_{i}-\bar{n}_{i}$ are replaced by spin half operators $S_{i}^{+}, S_{i}^{-}$, and $S_{i}^{z}+\frac{1}{2}$ respectively. This transformation maps (1) onto the quantum $S=\frac{1}{2} x x z$ Model [12]

$$
H^{x x z}=\sum_{\langle i j\rangle}\left(\frac{J^{z}}{S^{2}} S_{i}^{z} S_{j}^{z}+\frac{J}{2 S^{2}}\left(e^{i \theta_{i j}} S_{i}^{+} S_{j}^{-}+\text {H.c }\right)\right)
$$

where the Ising coupling is $J^{z}=V / 4$, and we limit our discussion to easy plane anisotropy $J^{z} \leq J$, in order to avoid the charge density wave phases [9]. A pure gauge transformation $\phi \rightarrow \phi+1$ on (11) or (2) leaves their spectrum invariant.

It is instructive to consider the classical (large $S$ ) ground state energies of (2) which are adiabatically connected to the ground states at $\phi=i, i=0,1, \ldots$, 


$$
E_{c l}^{i}(\phi)=J L_{y} L_{x} \delta n(1-\delta n) \cos \left(\frac{2 \pi(\phi-i)}{L_{x}}\right),
$$

where $\delta n \equiv n-\bar{n}$. At $\phi=q / 2, q$ integer, pairs of classical ground states of oppositely directed supercurrents become degenerate, as depicted in Fig. (1).

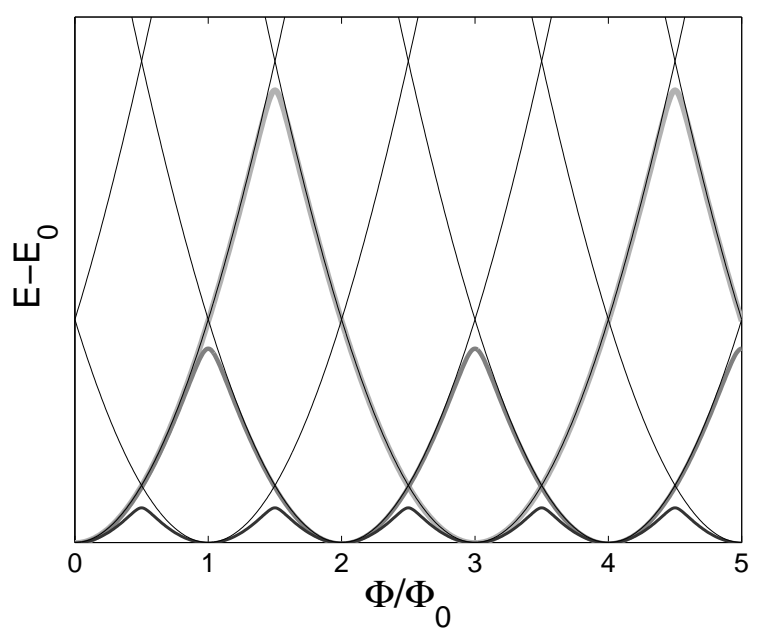

FIG. 1. Schematic adiabatic ground state energies as a function of Aharonov Bohm flux $E(\Phi)$, where $E_{0}=E(0)$. Thin lines: classical energies $E_{c l}^{i}(3)$. Thick lines: quantum adiabats of periods $q \Phi_{0}$, for $q=1,2,3$. Notice the level crossings for $q \neq 1$, which are protected by the selection rules (4).

Tunneling paths between the two classical ground states can be constructed as histories of vortices traversing the lattice in the $y$ direction, or nucleation and separation of a vortex-antivortex pairs upto the two edges. The tunneling matrix elements, unless prohibited by selection rules, open minigaps at the avoided level crossings. This allows their precise computation from the (many body) eigenenergies as a function of $\mathrm{AB}$ flux [14 16.

The Hamiltonian (2) was diagonalized using the Lanczos algorithm using lattice momentum and total magnetization to block-diagonalize the matrix. For the two leg ladder at $S_{\text {tot }}^{z}=0$, we find a regime of $J^{z}<J$ where the minigaps at $\phi=1 / 2$ remain finite as $L_{x}$ increases, which indicates a Haldane gap phase in the thermodynamic limit. This phase has been previously established for isotropic integer spin chains [17], and half odd integer ladders [18] and chains at finite magnetic fields [10].

To establish this phase we plot in Fig. 2, the magnon gap [19] at $\phi=1 / 2$ as a function of $1 / L_{x}$, and extrapolate the results to $1 / L_{x} \rightarrow 0$. However, for the extrapoltion to be justified, we must be certain that we have reached the asymptotic $L_{x}>>\xi$ regime where $\xi$ is the correlation length. $\xi$ was calculated from the superfluid stiffness $K=\partial^{2} E / \partial \phi^{2}$, which was found to fit to an exponential $K\left(L_{x}\right) \propto \exp \left(-L_{x} / \xi\right)$. We find that in the regime

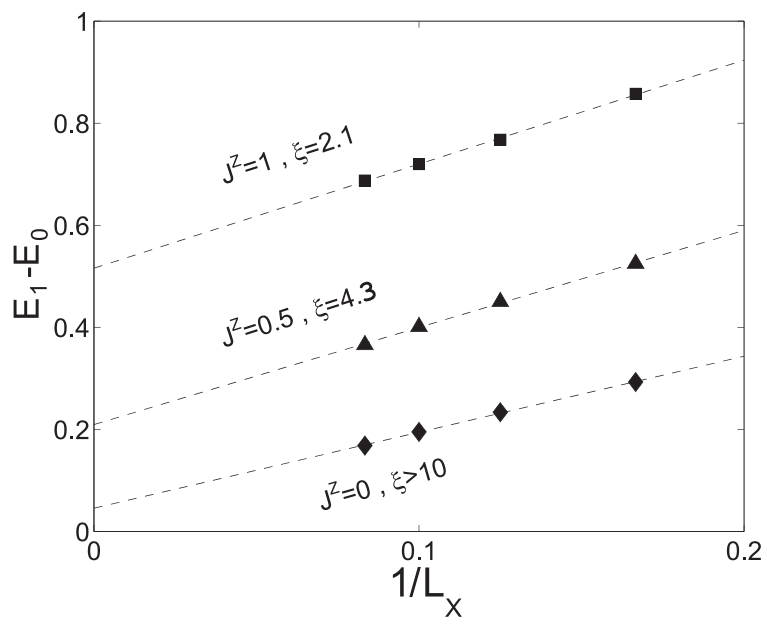

FIG. 2. Haldane gaps. Magnon excitation energies for two leg Josephson ladders for different anisotropy $J^{z} / J$. Here $J=1$ and $E_{m}$ is the lowest eigenenergy of magnetization $m$ at flux $\Phi=\Phi_{0} / 2 . \quad \xi$ is the stiffness correlation length given by finite size scaling of ladder lengths upto $L_{x}=12$.

$J^{z} / J^{x} \in(0.5,1)$, the correlation length is $\xi \in(4.26,2.13)$ which is safely smaller than the larger system dimensions. For the pure $x y$ model at $J^{z}=0$, however, $\xi$ reaches our largest system size. In this regime therefore, an extrapolated finite gap at $1 / L_{x}=0$ is not credible.

An easy-plane Haldane phase is explained as follows. The path integral of an even leg ladder of $S=1 / 2$ spins, can be mapped onto a classical partition function of an $\mathrm{O}(2)$ relativistic field theory in two dimensions [17, 13. Its temperature $T^{2 d}$ scales asymptotically as $\sim\left(S L_{y}\right)^{-1}$ 21]. This suggests that below a certain spin size and ladder width, a disordered phase with exponentially decaying correlations is possible, which translates into a finite gap for excitations and vanishing stiffness for long ladders. Above a critical width $L_{y}>L_{y}^{K T}$, correlations should decay as a power-law with a finite (1d) superfluid density $4 \pi^{2} \rho_{s}=\lim _{L_{x} \rightarrow \infty}\left(L_{x} K\right)>0$.

Selection rules for avoided level crossings. Vortex tunneling is enabled by the lattice since it breaks continuos translational symmetry. However, the remaining discrete translational symmetry imposes selection rules which are given by the following theorem.

Theorem: For the Hamiltonian (2), at $\phi=q / 2$, for integer $q$, any eigenstate $\left|S_{\text {tot }}^{z}, k_{x}, \alpha\right\rangle$ where $k_{x}$ is the lattice momentum in the $x$ direction, and $S_{\text {tot }}^{z}$ is the total magnetization, is at least two-fold degenerate unless the following condition is satisfied:

$$
\frac{S_{t o t}^{z}}{L_{x}}+\frac{k_{x}}{\pi}+S L_{y}=p / q(p, q \text { integers })
$$

The theorem is similar to Lieb, Shultz and Mattis (LSM) theorem [20] for half odd integer spin chains, and its extension to finite magnetizations by OYA [10]. Here, 
however, we prove exact degeneracies of the twisted $\mathrm{xxz}$ model on finite lattices, while previous work concerned gaplessness in the thermodynamic limit at zero external gauge field.

Before providing the proof, let us review three important classes to which the theorem for $S=1 / 2$ applies.

1. Odd ladders. $q=1, k_{x}=0, S_{\text {tot }}^{z}=0$. The selection rules (4) cannot be satisfied, implying exact ground state degeneracy at $\phi=1 / 2$. This is closely related to the existence of gapless excitations in the thermodynamic limit of short range half odd integer spin chains [20].

2. Even ladders with integer magnetization per rung. The selection rule is obeyed for $q=1$, which implies a minigap at the first avoided crossing of the ground states. If this minigap survives the $L_{x} \rightarrow \infty$ limit, the system is in the Haldane gap phase.

3. Even ladders with rational magnetization per rung. The selection rule is obeyed only for some $q>1$. This gives rise to a fractional $\mathrm{AB}$ periodicity of the ground state.

Proof: The twist operator is defined as

$$
\hat{O}(\phi) \equiv \exp \left(-i \frac{2 \pi}{L_{x}} \phi \sum_{\mathbf{r}} S^{z}(\mathbf{r}) x\right) .
$$

In addition, an $x$-inversion operator $I_{x}$ is defined $I_{x} S_{x, y}^{\alpha} I_{x}=S_{-x, y}^{\alpha}$. For any state $\psi_{0}=\left|S_{t o t}^{z}, k_{x}, \alpha\right\rangle$, we define the " $q$-conjugated" state $\psi_{q}$ as

$$
\left|\psi_{q}\right\rangle=\hat{O}(-q) I_{x}\left|\psi_{0}\right\rangle
$$

Lemma (degeneracy):

$$
\left\langle\psi_{q}|H(q / 2)| \psi_{q}\right\rangle=\left\langle\psi_{0}|H(q / 2)| \psi_{0}\right\rangle
$$

The Lemma is proven by a direct substitution of (6) in (7) noting that $O(\phi)$ is the explicit gauge transformation on $H$,

$$
H(\phi)=\hat{O}(-\phi) H(0) \hat{O}(\phi),
$$

and using the identities $I_{x} O(\phi) I_{x}=O(-\phi)$, and $\left[H(0), I_{x}\right]=0$.

The theorem is proved by showing that $\psi_{0}$ will be transformed by "q-conjugation" into an orthogonal state $\left\langle\psi_{q} \mid \psi_{0}\right\rangle=0$ unless the selection rule (雨) is obeyed. The unit lattice translation in the $x$ direction is $T_{x}$. We make use of the two identitites

$$
\begin{aligned}
T_{x} \hat{O}(-q) T_{x}^{-1} & =\exp \left(i 2 \pi q S_{\text {tot }}^{z} / L_{x}+i 2 \pi q S L_{y}\right) O(-q), \\
I_{x} T_{x} I_{x} & =T_{x}^{-1} .
\end{aligned}
$$

The lattice momentum of $\psi_{q}$ is given by

$$
T_{x}\left|\psi_{q}\right\rangle=\exp \left(-i \frac{2 \pi q}{L_{x}} S_{t o t}^{z}+i 2 \pi q S L_{y}+i k_{x}\right)\left|\psi_{q}\right\rangle
$$

It follows that $\left\langle\psi_{q} \mid \psi_{0}\right\rangle=0$ unless the momentum difference $\delta k_{x}=2 \pi\left(q S_{\text {tot }}^{z} / L_{x}-q S L_{y}\right)+2 k_{x}$ is an integer multiple of $2 \pi$, which proves selection rules (4). QED.

Translating back into the boson language, we consider the ground state at $\phi=0$, with $k_{x}=0$, and excess Bose density $\delta n=\frac{p}{q L_{y}}$. The selection rule implies that as $\phi$ is increased, the adiabatic ground state passes $q-$ 1 exact level crossings before reaching the first minigap allowed by (4). Hence it is clear that the ground state adiabatic periodicity in $\mathrm{AB}$ flux is $q \Phi_{0}$. Fig. 1 depicts the ground state evolution for the cases $q=1,2,3$. The critical current is bounded by

$$
I_{c r}(q) \leq(J / \hbar) L_{y} \delta n(1-\delta n) \sin \left(\frac{\pi}{L_{x}} q\right)
$$

which holds upto $q=L_{x} / 2$ where the bound coincides with the classical critical current $I_{c r}^{c l}=(J / \hbar) L_{y} \delta n(1-$ $\delta n)$.

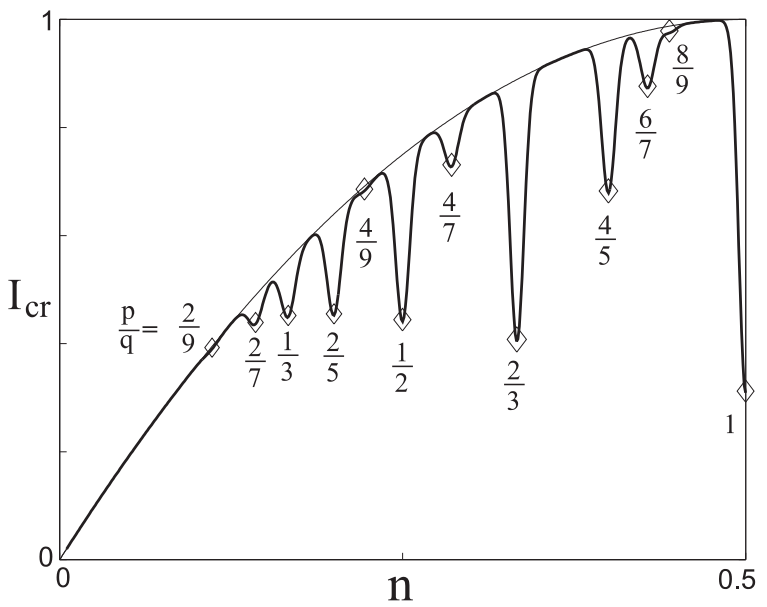

FIG. 3. Schematic diagram of critical currents versus Cooper pair density $n$ for an array of size $L_{x}=20$ and $L_{y}=2$. Rational numbers $p / q=n_{q} L_{y}$, which label the dips at $n_{q}$, are indicated. The classical critical current is depicted by a thin line. Above the critical currents at $n_{q}$, fractional AC Josephson frequencies are expected at $2 e V /(h q)$. Spatial disorder and finite temperature are introduced qualitatively by broadening of the dips.

In Fig. 3 the schematic structure of the critical current is plotted against the Cooper pairs density for a Josephson ladder with $L_{x}=20$. Notice the sharp dips in the critical current at rational densities which obey the selection rule. For weak easy plane anisotropy, these minima are expected to vanish in the thermodynamic limit, reflecting vanishing stiffness and gapped excitations at these points.

The classical Josephson frequency of an array of length $L_{x}$ array is $f_{c l}=2 e V /\left(h L_{x}\right)$, where $V$ is the total voltage drop in the $x$ direction. However at rational densities $n_{q}=p /\left(q L_{y}\right)$, for bias current slightly above $I_{c r}\left(n_{q}\right)$, an 
AC Josephson effect should be observed with frequency $f_{q}=2 e V /(q h)$. This could be pictured as current oscillations caused by moving on the adiabatic curves of Fig. 11. Alternatively, this effect could perhaps be better detected as fractional Shapiro steps in an external high frequency electromagnetic field 22].

Experimental realization. One of the experimental setups described in Ref. [4], has individual gate voltage probes which control the Cooper pair density at each island to high accuracy. The short range Bose Hubbard model for this type of JJA can be justified if the ratio of inter-island capacitance to gate capacitance obeys $\epsilon=C / C_{0}<<1$. This translates to onsite and intersite interactions of Eq. (11) given by $U=2 e^{2}(1-4 \epsilon) / C_{0}$, and $V=\epsilon 4 e^{2} / C_{0}$ respectively. $U>>V$ in this regime. In order map (11) to (2), we demand that $U>>J$, where $J$ is the Josephson coupling between islands. The easyplane regime is given by $V \leq 4 J$. As emphasized earlier, this inequality is crucial for eliminating possible charge density states, which may also exhibit reduced critical currents at commensurate fillings.

The junction parameters reported in Ref. [1] were $C_{0}=0.64 \mathrm{fF}$, and $C=1.0 \mathrm{fF}$, and $J=0.63^{\circ} \mathrm{K}$. To reach the desired regime, these parameters should be modified to approximately $C_{0}=3.0 \mathrm{fF}$, and $C=0.3 \mathrm{fF}$, and $J=0.2^{\circ} \mathrm{K}$.

Acknowlegements: Useful discussions with J. Avron, A. Stern, A. van Oudenaarden, and S-C. Zhang are gratefully acknowledged. The authors thank the Department of Physics, Stanford University, where part of this work was performed. AA acknowledges a grant from the Israel Science Foundation.

[1] S. Doniach, Phys. Rev. B24, 5063 (1981).

[2] D.B. Haviland, Y. Liu and A.M. Goldman, Phys. Rev. Lett. 62, 2180 (1989); A.F. Hebard and M.A. Paalanen, Phys. Rev. Lett. 65, 927 (1990); A. Yazdani and A. Kapitulnik, Phys. Rev. Lett. 34, 3037 (1995).

[3] P. Delsing, C.D. Chen, D.B. Haviland, Y. Harada and T.Claeson, Phys. Rev. B50, 3959 (1994).

[4] A. van Oudenaarden and J.E. Mooij, Phys. Rev. Lett. 76, 4947 (1996); A. van Oudenaarden, Ph.D. Thesis, (Delft University Press, 1998)

[5] M.P.A. Fisher, P.B. Weichman, G. Grinstein and D.S. Fisher, Phys. Rev. B40, 546 (89); C. Bruder, R. Fazio, A. Kampf, A. van Otterlo and G. Shon, Phys. Scr. 42, 159 (1992); R.T. Scalettar, G.G. Batrouni, A.P. Kampf and G.T. Zimanyi, Phys. Rev. B51, 8467 (95).

[6] G. E. Volovik, JETP Lett. 15, 81 (1972); P. Ao and D. J. Thouless, Phys. Rev. Lett. 72, 132 (1994); D. P. Arovas and J. A. Freire, Phys. Rev. B 55, 1068 (1997).

[7] M.P.A. Fisher and D.H. Lee, Phys. Rev. B39, 2756 (1989).

[8] A. Stern, Phys. Rev. B50, 10092 (1994).

[9] T. Matsubara and H. Matsuda, Prog. Theor. Phys. 16
569 (1956); K.S. Liu and M.E. Fisher, J. Low. Temp. Phys. 10, 655 (1973); A. Aharony and A. Auerbach Phys. Rev. Lett. 70, 1874 (1993).

[10] M. Oshikawa, M. Yamanaka, I. Affleck, Phys. Rev. Lett. 78, 1984 (1997).

[11] While (11) is sometimes used as a coarse grained theory of homogenous superconductors, it does not contain the fermion (pair breaking) excitations.

[12] For bipartite lattices, we replace the negative Bose hopping by a positive $x y$ exchange using a sublattice rotation [13.

[13] A. Auerbach, "Interacting Electrons and Quantum Magnetism", (Springer-Verlag. NY), Ch. 3; Chia Laguna lecture notes, cond-mat/9801294.

[14] R. Tao and F.D.M. Haldane, Phys. Rev. B33, 3844 (1986).

[15] Y. Gefen and D. J. Thouless, Phys. Rev. B47, 10423 (1993).

[16] A. Auerbach, Phys. Rev. Lett. 80, 817 (1998).

[17] F.D.M. Haldane, Phys. Lett. A93, 464 (1983).

[18] D.V. Khveshchenko, Phys. Rev. B 50, 380 (1994); E. Dagotto and T.M. Rice, Science 271, 618 (1996); G. Sierra, Jour. Phys. A 29, 3299 (1996).

[19] The minigaps of Fig. 1 are between $S_{t o t}^{z}=0$ states, while magnons of $S_{t o t}^{z}= \pm 1$, have lower excitation energies due to easy plane anisotropy $J^{z}<J$.

[20] E. Lieb, T. Schultz, D. Mattis, Ann. Phys. (N.Y.) 16, 407 (1961). I. Affleck and E.H. Lieb, Lett. Math. Phys. 12, 57 (1986); I. Affleck, Phys. Rev. B, 37, 5186 (1988).

[21] Higher order corrections were obtained for the Heisenberg case by S. Chakravarty, Phys. Rev. Lett., 774446 (1996).

[22] L.L. Sohn and M. Octavio, Phys. Rev. B 49, 9236 (1994); 